\title{
Exchange transfusion safety and outcomes in neonatal hyperbilirubinemia
}

\author{
Mattie F. Wolf ${ }^{1} \cdot$ Julie Childers $^{1} \cdot$ Keyaria D. Gray ${ }^{1} \cdot$ Caroline Chivily $^{2} \cdot$ Mike Glenn $^{2} \cdot$ Laila Jones $^{2} \cdot$ Mini Kpa $^{2} \cdot$

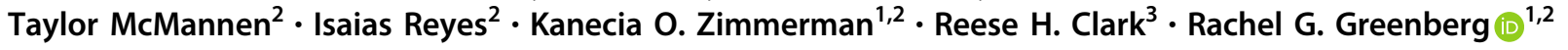

Received: 10 October 2019 / Revised: 11 February 2020 / Accepted: 25 February 2020 / Published online: 9 March 2020

(c) The Author(s), under exclusive licence to Springer Nature America, Inc. 2020

\begin{abstract}
Objective To characterize the prevalence of exchange transfusion (ET), clinical characteristics of infants receiving ET, and ET-associated morbidity and mortality.

Study design We conducted a multicenter cohort study of infants $\geq 23$ weeks of gestational age (GA) with hyperbilirubinemia who underwent ET within 30 days of birth from 1997 to 2016 . We examined clinical characteristics and adverse events after ET. We used multivariable logistic regression to examine the association between clinical risk factors and death. Result A total of 1252 infants were included; 4\% died within 7 days of ET and $6 \%$ died before discharge. Compared with infants $\geq 37$ weeks of GA, infants $\leq 29$ weeks of GA had greater odds of death (adjusted odds ratio [95\% confidence interval $]=20.08[7.32,55.07])$.

Conclusions Infants $\leq 29$ weeks of GA had greater odds of death following ET compared with term infants. These data will support clinicians in evaluating risks and prognosis for infants who require ET.
\end{abstract}

\section{Introduction}

Hyperbilirubinemia affects upto $85 \%$ of neonates born at term gestation ( $\geq 37$ weeks gestational age [GA]) and $80 \%$ of premature newborns [1-3]. While severe hyperbilirubinemia (total serum bilirubin [TSB] level of $>20 \mathrm{mg} / \mathrm{dL}$ [342.1 $\mu \mathrm{mol} / \mathrm{L}]$ ) occurs in $<2 \%$ of term infants, it can lead to permanent neurodevelopmental delay and kernicterus (i.e., chronic bilirubin encephalopathy) [2]. Furthermore, though preventable, kernicterus occurs in $20 \%$ of infants with TSB $>30 \mathrm{mg} / \mathrm{dL}[4,5]$. The prevalence of kernicterus on autopsy in preterm infants who die is thought to be around 4\% [6]. In addition, although small case series have described survivors with neurologic sequelae associated with

Rachel G. Greenberg

rachel.greenberg@duke.edu

1 Department of Pediatrics, Duke University School of Medicine, Durham, NC, USA

2 Duke Clinical Research Institute, Duke University School of Medicine, Durham, NC, USA

3 Pediatrix Medical Group, Sunrise, FL, USA hyperbilirubinemia in term infants [7, 8], there are no data on the prevalence of kernicterus in preterm survivors.

Kernicterus is preventable through the use of phototherapy, treatment with intravenous immunoglobulins (IVIg), or the use of exchange transfusion (ET) to lower serum bilirubin levels [9]. Phototherapy is an effective, noninvasive, first-line treatment to lower the levels of unconjugated bilirubin. IVIg has some support as an adjuvant therapy for hyperbilirubinemia in cases of Rh hemolytic disease, although the efficacy is controversial [10]. ET is more efficient at reducing bilirubin levels and is often used when maximal phototherapy and/or IVIg are unsuccessful or when hemolysis is excessive [11, 12]; however, ET has known complications including vascular accidents, cardiovascular compromise, and electrolyte and hematologic derangement [13]. Furthermore, infants undergoing ET are at higher risk of requiring intubation and mechanical ventilation, both of which are associated with additional complications [14].

The incidence of meeting or exceeding American Academy of Pediatrics thresholds for ET is low, involving $\sim 1.2$ per 1000 live births. Nevertheless, premature infants have a tenfold increased risk of eventual bilirubin level meeting or exceeding thresholds for ET compared with term neonates [15]. Furthermore, the frequency of ET has 
steadily declined over time due to increased hyperbilirubinemia screening, treating pregnant women who are Rh-negative with Rh-factor therapy, the increased use of phototherapy, development of IVIg, and ET safety concerns $[4,16]$. Previous studies evaluating safety do not have the most current data and have been single-center evaluations or included only relatively small neonatal populations [13, 14, 16-19]. In this cohort study, we sought to further assess and characterize the prevalence of ET use in our neonatal intensive care unit (NICU) population, as well as the safety of ET for treating hyperbilirubinemia.

\section{Methods}

\section{Study design}

We conducted a retrospective cohort study of infants $\geq$ 23 weeks of GA discharged from 1997 to 2016 from Pediatrix Medical Group NICUs. For the ET population, we included infants with hyperbilirubinemia who underwent ET within 30 days of birth. Demographic, clinical, and maternal data were extracted from a clinical data warehouse that prospectively captures data from electronic health records, including daily progress notes and other documentation generated by clinicians using a computerassisted tool [20]. The study was approved by the Duke University Institutional Review Board as exempt research.

\section{Variables of interest}

We defined ET as any infant who received ET. Known partial volume ETs were excluded. Hyperbilirubinemia was defined as either (1) a documented diagnosis of hyperbilirubinemia or (2) a serum bilirubin level $>15 \mathrm{mg} / \mathrm{dL}$ for infants 38 weeks gestation and older, or $>10 \mathrm{mg} / \mathrm{dL}$ in infants aged $\leq 37$ weeks prior to or on the day of ET, without evidence of an alternate diagnosis that may have justified a requirement for ET [21, 22]. We also evaluated maximum bilirubin levels for infants 1 day following ET and 7 days after ET, and we noted the use of IVIg prior to ET. Small for gestational age (SGA) status was defined based on the Olsen definition [23]. The number of ETs was evaluated as a percentage of total infants over time during the study period.

Laboratory abnormalities evaluated 1 day prior to, 1 day after, and within 7 days after ET included: thrombocytopenia (platelets $<100,000 / \mu \mathrm{L}[24,25]$ ), hypocalcemia (total serum calcium level $<7 \mathrm{mg} / \mathrm{dL}$ [26]), and hyperkalemia (potassium $>7 \mathrm{mEq} / \mathrm{L}$ [27]). Our database did not allow us to distinguish timing of laboratory values, so we could not determine whether laboratory values on the day of ET occurred before or after the ET. As a sensitivity analysis to ensure we did not miss important abnormalities, we evaluated the proportion of abnormal laboratory values on the day of ET in infants who were missing values within 7 days after ET. Severe intraventricular hemorrhage (IVH) included grades III and IV [28]. We defined neonatal sepsis as any positive blood or cerebrospinal fluid culture, including definite and probable coagulase-negative staphylococcal at any point prior to, on 1 day after, and within 7 days after ET [29]. Necrotizing enterocolitis (NEC) included diagnosis of stage 2 or 3 by the Bell criteria [30]. Seizures were defined as documented by the provider. IVH, sepsis, NEC, and seizures were evaluated at any point prior to, on 1 day after, and within 7 days after ET. Respiratory support was collected on the day of, 1 day after, and within 7 days after ET and included nasal cannula, continuous positive airway pressure, and mechanical ventilation as documented by the provider. With the exception of laboratory abnormalities and sepsis, events were only reported after ET if they were not present prior to ET. Laboratory abnormalities and respiratory support were reported among infants who had evaluations (nonmissing data) within the time period of interest.

\section{Statistical analysis}

We used descriptive statistics, including counts, percentages, medians, and percentiles, to summarize demographic characteristics and clinical data. Among the entire population of infants $\geq 23$ weeks of GA, we determined the overall prevalence of ET in the setting of hyperbilirubinemia in the first 30 postnatal days by discharge year. Among the ET analysis population, clinical characteristics of interest included postnatal age at the time of ET and at discharge, the number of ETs, and receipt of IVIg prior to ET. We reported the presence of the above clinical and laboratory events of interest prior to and after ET. We reported the percentage of infants who died of any cause within 7 days following ET and during hospitalization overall and by GA. We also reported the percentage of infants who experienced grade 3 or 4 IVH, sepsis, NEC, thrombocytopenia, hypocalcemia, hyperkalemia, and seizures within 7 days following ET by GA. We used multivariable logistic regression to examine the association between death before discharge and GA, SGA status, and maximum total serum bilirubin. STATA 15.1 (College Station, TX) was used to perform statistical analyses. A $p$ value of $<0.05$ was considered statistically significant.

\section{Results}

Of a total $1,247,425$ infants $\geq 23$ weeks of GA from 392 sites, $1252(0.1 \%)$ underwent ET. Overall, the number 


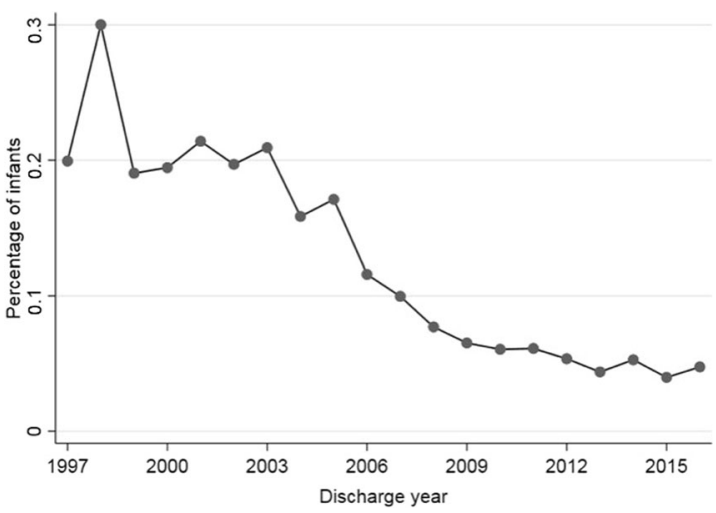

Fig. 1 Percentage of infants undergoing ET over time. This figure displays the percentage of infants who have undergone ET from 1997 to 2015. ET exchange transfusion.

of infants receiving ET decreased from a maximum of $0.3 \%$ to a minimum of $0.05 \%$ of all infants $\geq 23$ weeks in our study population over the course of the study period (Fig. 1). Among the infants who underwent ET, the median (25th-75th percentile) GA and birthweight of infants receiving ET were 37 weeks (33-38 weeks) and $2789 \mathrm{~g}$ (1928-3275 g), respectively. A slight majority of infants were male $(55 \%)$, and a plurality had White race $(45 \%)$ (Table 1). The majority (91\% [1134/1252]) of the study population received only one ET, and $14 \%$ of the study population (176/1252) received IVIg prior to ET (Table 1). The trend of IVIg utilization increased over time; incidence did not exceed $7 \%$ in the first 6 years of study, then increased to between 12 and $23 \%$ over the subsequent 7 years, followed by an incidence of $30 \%$ or greater in 4 of the 5 final years of study. The median postnatal age at the time of ET was 1 day ( 0 and 3 days) with more than onequarter of infants receiving an ET on the day of birth; median postnatal age at discharge was 11 days (7 and 24).

There was a slight decrease in median bilirubin level $(\mathrm{mg} / \mathrm{dL})$ after ET, with stable levels between 1 and 7 days following ET (Table 2). Less than half of the infants received respiratory support prior to ET (46\% [566/1240]), and of those not receiving respiratory support, 69/676 (10\%) received respiratory support within 7 days after ET. While a relatively large proportion of infants received mechanical ventilation prior to receiving an ET (23\% [282/ 1252]), few of those not receiving ventilation began receiving mechanical ventilation within seven days post ET (3\% [32/950]). The most common adverse effect of ET was thrombocytopenia at 7 days following ET, which affected $64 \%(646 / 1013)$ of our study population. Of 52 infants without platelet evaluation within 7 days who had platelet evaluation on the day of ET, 7 (13\%) had thrombocytopenia on the day of ET. Hyperkalemia affected 5\% (46/886) by 7 days after ET. Of 48 infants without potassium evaluation within 7 days who had potassium evaluation on the day of
Table 1 Demographic characteristics of infants undergoing exchange transfusion.

\begin{tabular}{lc}
\hline Characteristic & $N=1252(\%)$ \\
\hline Gestational age (weeks) & \\
$\quad \leq 29$ & $159(13)$ \\
$30-34$ & $272(22)$ \\
$35-37$ & $338(27)$ \\
$>37$ & $483(39)$ \\
Birthweight (g) & \\
$<1000$ & $101(8)$ \\
$1000-1499$ & $109(9)$ \\
$1500-2499$ & $284(23)$ \\
$>2499$ & $758(61)$ \\
Male & $687(55)$ \\
SGA & $134(11)$ \\
Race/ethnicity & \\
White & $540(45)$ \\
Black & $263(22)$ \\
Hispanic & $295(25)$ \\
Other & $93(8)$ \\
Inborn & $856(69)$ \\
Number of exchange transfusions & \\
1 & $1134(91)$ \\
2 & $104(8)$ \\
IVIg prior to exchange transfusion & $14(1)$ \\
\hline & $176(14)$ \\
\hline &
\end{tabular}

IVIg intravenous immunoglobulin, SGA small for gestational age.

ET, $3(6 \%)$ had hyperkalemia on the day of ET. Hypocalcemia was not detected prior to ET, but appeared in $2 \%$ $(19 / 818)$ of infants whose calcium was measured within 7 days following ET. Of 75 infants without calcium evaluation within 7 days who had calcium evaluation on the day of ET, 5 (7\%) had hypocalcemia on the day of ET. Prior to ET, sepsis occurred in 2\% (31/1252) of patients, while within 7 days after ET, only $0.6 \%$ developed a new episode sepsis (7/1252). The incidence of NEC was $<1 \%$ at all time periods evaluated. The incidence of seizures was documented to be similar at $2 \%$ prior to ET and $2 \%$ on 7 days after ET (Table 2).

Hospital mortality could be determined for $1161 / 1252$ (93\%) infants. A total of $4 \%$ (42/1161) of infants receiving ET died within 7 days following ET; overall hospital mortality was $6 \%(65 / 1161)$. Infants $\leq 29$ weeks of GA had the highest mortality within 7 days after ET, 17\% (24/141). They also had the highest overall hospital mortality of $27 \%$ (38/141) (Table 3). Of the infants who died during hospitalization, none developed NEC, two developed seizures, and five developed IVH within the 7 days post ET. On adjusted analysis, there was no significant association between death during hospitalization and maximum TSB 
Table 2 Clinical and laboratory characteristics of infants undergoing exchange transfusion.

\begin{tabular}{|c|c|c|c|}
\hline & Prior to ET & 1 day after ET & 7 days after ET \\
\hline Bilirubin level $^{\mathrm{a}}$, median (25th-75th percentile) & $18(12,22)$ & $14(11,18)$ & $15(11,18)$ \\
\hline GA $\leq 29$ weeks & $12(9,16)$ & $9(6,13)$ & $10(7,13)$ \\
\hline GA 30-34 weeks & $14(10,20)$ & $14(10,18)$ & $15(11,18)$ \\
\hline GA $35-37$ weeks & $18(13,23)$ & $15(11,18)$ & $16(13,19)$ \\
\hline GA $>37$ weeks & $20(16,24)$ & $15(12,19)$ & $16(12,19)$ \\
\hline Respiratory support ${ }^{\mathrm{b}}, n / N(\%)$ & $566 / 1240(46)$ & $41 / 674(6)$ & $69 / 676(10)$ \\
\hline Intubation, $n / N(\%)$ & $282 / 1240(23)$ & $19 / 950(2)$ & $32 / 950(3)$ \\
\hline Grades III and IV IVH, $n / N(\%)$ & $26 / 1252(2)$ & $5 / 1226(0.4)$ & $13 / 1226(1)$ \\
\hline Sepsis, $n / N(\%)$ & $31 / 1252(2)$ & $3 / 1252(0.2)$ & $7 / 1252(0.6)$ \\
\hline Necrotizing enterocolitis, $n / N(\%)$ & $7 / 1252(0.6)$ & $2 / 1245(0.2)$ & $5 / 1245(0.4)$ \\
\hline Thrombocytopenia $(<100,000 / \mathrm{mL}), n / N(\%)$ & $64 / 348(18)$ & $413 / 895(46)$ & $646 / 1013(64)$ \\
\hline Hypocalcemia $^{\mathrm{c}}(<4.2 \mathrm{mg} / \mathrm{dL}), n / N(\%)$ & $0 / 177$ & $9 / 670(1)$ & $19 / 818(2)$ \\
\hline Hyperkalemia $^{\mathrm{c}}(>7 \mathrm{mEq} / \mathrm{L}), n / N(\%)$ & $6 / 202(3)$ & $21 / 747(3)$ & $46 / 886(5)$ \\
\hline Seizures, $n / N(\%)$ & $22 / 1252(2)$ & $7 / 1230(0.6)$ & $22 / 1230(2)$ \\
\hline
\end{tabular}

ET exchange transfusion, GA gestational age.

aBilirubin level for "prior to ET" is from the day of ET.

bIncludes nasal cannula, continuous positive airway pressure, or ventilator.

"Laboratory values other than bilirubin for "prior to ET" were obtained 1-2 days prior to ET.

Table 3 Laboratory and clinical outcomes associated with exchange transfusion by gestational age.

\begin{tabular}{|c|c|c|c|c|}
\hline & \multicolumn{4}{|c|}{ Gestational age (weeks) } \\
\hline & $\leq 29$ & $30-34$ & $35-37$ & $>37$ \\
\hline Death within 7 days of ET & $24 / 141(17)$ & $10 / 252(4)$ & $3 / 311(1)$ & $5 / 457(1)$ \\
\hline Death within hospitalization & $38 / 141(27)$ & $14 / 252(6)$ & $7 / 311(2)$ & $6 / 457(1)$ \\
\hline Grade III and IV IVH within 7 days of ET & $9 / 138(7)$ & $4 / 268(1)$ & $0 / 338(0)$ & $0 / 482(0)$ \\
\hline Sepsis within 7 days of ET & $5 / 159(3)$ & $0 / 272(0)$ & $2 / 338(0.6)$ & $0 / 483(0)$ \\
\hline Necrotizing enterocolitis within 7 days of ET & 2/157 (1) & $2 / 268(0.8)$ & $0 / 338(0)$ & $1 / 482(0.2)$ \\
\hline Thrombocytopenia $(<100,000 / \mathrm{mL})$ within 7 days of ET & $96 / 141(68)$ & $157 / 236(67)$ & $161 / 268(60)$ & $232 / 368(63)$ \\
\hline Hypocalcemia $(<4.2 \mathrm{mg} / \mathrm{dL})$ within 7 days of ET & $7 / 123(6)$ & $4 / 206(2)$ & $4 / 215(2)$ & $4 / 274(1)$ \\
\hline Hyperkalemia $(>7 \mathrm{mEq} / \mathrm{L})$ within 7 days of ET & $15 / 126(12)$ & $10 / 219(5)$ & $10 / 233(4)$ & $11 / 308(4)$ \\
\hline Seizures within 7 days of ET & $5 / 152(3)$ & $4 / 269(1)$ & $5 / 332(2)$ & $8 / 477(2)$ \\
\hline
\end{tabular}

Data are given as $n / N(\%)$.

ET exchange transfusion, $I V H$ intraventricular hemorrhage.

(odds ratio $[\mathrm{OR}]=0.95,[0.90,1.00] ; p=0.05$ ) or SGA status $(\mathrm{OR}=2.07,[0.99,4.31] ; p=0.05)$. Compared with infants $>37$ weeks of GA, infants $\leq 29$ weeks of GA $(\mathrm{OR}=$ $20.08,[7.32,55.07] ; p<0.001)$ and 30-34 weeks of GA $(\mathrm{OR}=3.31, \quad[1.12, \quad 9.79] ; \quad p=0.03)$ had significantly increased odds of death (Table 4).

\section{Comments}

In this large cohort of $>1200$ preterm and term infants who received ET for hyperbilirubinemia, we found that infants < 35 weeks of GA, and especially those $\leq 29$ weeks of GA, were at a higher risk of death following ET compared to term infants. We also evaluated use of IVIg prior to ET and adverse events by GA surrounding ET, which has not been previously reported. Only $14 \%$ of infants received IVIg within our study population, with increasing incidence over time. One prior retrospective study found that only 6 of its 107 participants $(6 \%)$ received IVIg prior to ET, demonstrating a similar pattern of use to the early years of our study [16]. Interestingly, a 2018 Cochrane review of nine studies with 658 infants examining the efficacy of IVIg in preventing ET did not show sufficient benefit to warrant a recommendation for systematic use of IVIg as a primary intervention for alloimmune hemolytic disease of the newborn to prevent ET [31]. Nevertheless, the increasing incidence of IVIg utilization may indicate improved ability to identify those infants in whom IVIg may provide benefit. 
Table 4 Multivariable logistic regression to evaluate association between risk factors and hospital death for infants undergoing exchange transfusion.

\begin{tabular}{llc}
\hline Risk factor & Adjusted OR (95\% CI) & $p$ value \\
\hline $\begin{array}{l}\text { Gestational age (weeks) } \\
\leq 29\end{array}$ & $20.08(7.32-55.07)$ & $<0.001$ \\
$30-34$ & $3.31(1.12-9.79)$ & 0.03 \\
$35-37$ & $1.50(0.43-5.29)$ & 0.52 \\
$>37$ & {$[$ Ref] } & \\
Small for gestational age & $2.07(0.99-4.31)$ & 0.05 \\
$\begin{array}{l}\text { Maximum total serum bilirubin } \\
\text { (mg/dL) }\end{array}$ & $0.95(0.90-1.00)$ & 0.05 \\
\hline
\end{tabular}

$C I$ confidence interval, $O R$ odds ratio.

Thrombocytopenia was the most common laboratory adverse event we observed in this cohort, while hypocalcemia and hypokalemia were less common. Hakan et al. [16] and Steiner et al. [17] conducted retrospective studies examining the adverse events associated with ET in 107 and 306 infants, respectively. These studies found thrombocytopenia, hypocalcemia, and hypokalemia to be among the most common adverse outcomes, which were defined in both studies as occurring within 7 days after ET [16, 17]. Furthermore, in a study by Sabzehei et al. [17] that examined 148 infants who received ET, the authors found thrombocytopenia in $26(17.6 \%)$ and hypocalcemia in 17 $(11.5 \%)$ neonates [19]. These studies are significantly smaller than our current study and looked at only one time point following ET. We described these adverse effects at two different time points following ET to provide a more accurate timeline of when these conditions may occur; this timeline may inform medical providers regarding improved treatment and/or prevention strategies. There were also rarer adverse events, such as need for respiratory support, sepsis, and NEC. The association between ET and NEC has been previously reported in the literature and ranges from $0.9 \%$ $(1 / 106)$ to $1.3 \%$ (8/614) of infants undergoing ET in multiple retrospective studies completed by chart review [32, 33], though another retrospective study with fewer infants $(n=55)$ did not observe this association [18]. An association between NEC and IVIg has been reported [34].

Adverse neurological outcomes, such as IVH and seizures, occur in infants following ET. Although few studies have explored the incidence of these outcomes, a retrospective study by Patra et al. found that among a group of 66 preterm and term infants who received ET, only one infant experienced a seizure [18]. In our larger study, $2 \%$ developed seizures, and 1\% developed IVH within 7 days after ET. Not surprisingly, these diagnoses were most common in the most premature infants. The difference in these findings is most likely due to variation in the size of study populations. Although these neurological events were not the most common adverse outcomes, they have the possibility to produce long-term effects and should be considered when assessing risk of ET. Our study is limited in that we do not have data on long-term neurodevelopmental outcomes.

Finally, mortality continues to be a concern for infants receiving ET. Although the overall hospital mortality was low, when comparing mortality by GA, infants $<35$ weeks of GA, and especially those $\leq 29$ weeks of GA, were at a significantly higher risk of death after ET. Preterm infants may be at higher risk for death associated with ET because they are more likely to experience poor health and a greater number of comorbidities leading up to a need for ET. Furthermore, transfusions of any kind can put them at risk for NEC and/or severe IVH that may lead to seizure [35]. The odds of death for an infant receiving ET was slightly lower with higher levels of maximum TSB, which was unexpected, given our knowledge of indications for ET. While our study produced these surprising results, additional information regarding the rate of bilirubin rise along with the bilirubin by hour of life and by GA may provide more insight. Unfortunately, time of bilirubin levels was not available from our dataset. While our dataset did not include cause of death, a recent study discussed how the risks for death are mostly due to complications prior to ET and that none died due to ET itself. Sabzehei et al. [17] conducted a 5-year retrospective study, which was shorter than our study, examining the outcomes of 148 infants that received ET [19]. Much like our study design, Sabzehei et al. [17] included many similar outcomes, such as thrombocytopenia, hypocalcemia, hypokalemia, NEC, and cardiovascular support, but the ways in which the investigators reported death differed from our methods. Sabzehei et al. [17] reported death by GA, but only differentiated between preterm $(0 \%)$ and term infants $(1 \%)$, whereas we separated death by several categories of GA [19]. Furthermore, our study made the distinction between death within 7 days of ET (4\%) and death during hospitalization (6\%). The differences in death observed in these two studies can likely be attributed to study size, since our study examined a much larger patient population. Finally, we found that infants who were SGA had a trend toward higher odds of hospital mortality, although this was not statistically significant. This finding is similar to prior studies and may be attributable to increased risks of respiratory distress syndrome, NEC, lateonset sepsis, severe retinopathy of prematurity, and chronic lung disease [36].

Our study had several limitations. First, due to the retrospective nature of this study, we could not randomize those who received ET, nor could we control for differences in clinical practice or how diagnoses were documented by the provider. Second, we could not account for the cause of cardiovascular support or intubation, nor could be account 
for the causes of jaundice within our selected population. Third, we do not have the exact time of ET documented, so it is unclear whether the lab studies were obtained before, during, or after transfusion on the particular day that ET was performed. Whether there were practice differences is also unclear, including use of citrate for ET or a protocol for calcium repletion that may have accounted for the low rate of hypocalcemia. Finally, because infants did not have laboratory testing every day, variable numbers of infants could be evaluated for adverse events surrounding ET at different time points (Table 2). Despite these limitations, there are a number of strengths that enhance the overall validity of our study. Access to the Pediatrix Clinical Data Warehouse provided a large, diverse, and more globally representative study group, as well as a long study period; access to this data permitted us to quantify adverse outcomes and death by such measures as GA and days after ET.

In conclusion, infants $\leq 29$ weeks of GA had significantly greater odds of death following ET compared with term infants, but higher maximum TSB was not significantly associated with increased death. Adverse events, such as thrombocytopenia, hypocalcemia, and hypokalemia, were most commonly observed around the time of ET, and neurological effects, such as IVH and seizures, were less common. These data will support clinicians in evaluating risks and prognosis for infants in the NICU who require ET.

Acknowledgements PTN Steering Committee Members: Daniel K. Benjamin Jr., Christoph Hornik, Kanecia Zimmerman, Phyllis Kennel, and Rose Beci, Duke Clinical Research Institute, Durham, NC; Chi Dang Hornik, Duke University Medical Center, Durham, NC; Gregory L. Kearns, Scottsdale, AZ; Matthew Laughon, University of North Carolina at Chapel Hill, Chapel Hill, NC; Ian M. Paul, Penn State College of Medicine, Hershey, PA; Janice Sullivan, University of Louisville, Louisville, KY; Kelly Wade, Children's Hospital of Philadelphia, Philadelphia, PA; Paula Delmore, Wichita Medical Research and Education Foundation, Wichita, KS. The Eunice Kennedy Shriver National Institute of Child Health and Human Development (NICHD): Perdita Taylor-Zapata and June Lee. The Emmes Company, LLC (Data Coordinating Center): Ravinder Anand, Gaurav Sharma, Gina Simone, Kim Kaneshige, and Lawrence Taylor. PTN Publications Committee: Chaired by Thomas Green, Ann \& Robert H. Lurie Children's Hospital of Chicago, Chicago, IL

Funding This work was funded under the National Institute of Child Health and Human Development (NICHD) contract (HHSN275201000003I) for the Pediatric Trials Network (PI Danny Benjamin). The content is solely the responsibility of the authors and does not necessarily represent the official views of the National Institutes of Health. This work was also supported by Duke Clinical Research Institute's R25 Summer Training in Academic Research (STAR) Program (grant \#5R25HD076475-07).

\section{Compliance with ethical standards}

Conflict of interest RGG has received support from industry for research services (https://dcri.org/about-us/conflict-of-interest/). The authors have no other conflicts of interest relevant to this article to disclose.

Publisher's note Springer Nature remains neutral with regard to jurisdictional claims in published maps and institutional affiliations.

\section{References}

1. Bhutani VK, Stark AR, Lazzeroni LC, Poland R, Gourley GR, Kazmierczak S, et al. Predischarge screening for severe neonatal hyperbilirubinemia identifies infants who need phototherapy. J Pediatr. 2013;162:477-82. e1

2. Sgro M, Campbell D, Shah V. Incidence and causes of severe neonatal hyperbilirubinemia in Canada. CMAJ. 2006;175:587-90.

3. Truman P. Jaundice in the preterm infant. Paediatr Nurs. 2006;18:20-2.

4. Bhutani VK, Johnson L. Kernicterus in the 21st century: frequently asked questions. J Perinatol. 2009;29(Suppl 1):S20-4.

5. Bhutani VK, Johnson L. Synopsis report from the pilot USA Kernicterus Registry. J Perinatol. 2009;29(Suppl 1):S4-7.

6. Watchko JF, Claassen D. Kernicterus in premature infants: current prevalence and relationship to NICHD Phototherapy Study exchange criteria. Pediatrics. 1994;93:996-9.

7. Watchko JF, Maisels MJ. Jaundice in low birthweight infants: pathobiology and outcome. Arch Dis Child Fetal Neonatal Ed. 2003;88:F455-8.

8. Okumura A, Kidokoro H, Shoji H, Nakazawa T, Mimaki M, Fujii $\mathrm{K}$, et al. Kernicterus in preterm infants. Pediatrics. 2009;123: e1052-8.

9. Brown AK, Kim MH, Wu PY, Bryla DA. Efficacy of phototherapy in prevention and management of neonatal hyperbilirubinemia. Pediatrics. 1985;75:393-400.

10. Nasseri F, Mamouri GA, Babaei H. Intravenous immunoglobulin in $\mathrm{ABO}$ and $\mathrm{Rh}$ hemolytic diseases of newborn. Saudi Med J. 2006;27:1827-30.

11. Newman TB, Maisels MJ. Evaluation and treatment of jaundice in the term newborn: a kinder, gentler approach. Pediatrics. 1992;89:809-18.

12. No authors listed. Practice parameter: management of hyperbilirubinemia in the healthy term newborn. American Academy of Pediatrics. Provisional Committee for Quality Improvement and Subcommittee on Hyperbilirubinemia. Pediatrics. 1994;94:558-65.

13. Ives NK. Management of neonatal jaundice. Paediatr Child Health. 2011;21:270-6.

14. Chitty HE, Ziegler N, Savoia H, Doyle LW, Fox LM. Neonatal exchange transfusions in the 21 st century: a single hospital study. J Paediatr Child Heal. 2013;49:825-32.

15. Flaherman VJ, Kuzniewicz MW, Escobar GJ, Newman TB. Total serum bilirubin exceeding exchange transfusion thresholds in the setting of universal screening. J Pediatr. 2012;160:796-800. e1

16. Hakan N, Zenciroglu A, Aydin M, Okumus N, Dursun A, Dilli D. Exchange transfusion for neonatal hyperbilirubinemia: an 8-year single center experience at a tertiary neonatal intensive care unit in Turkey. J Matern Neonatal Med. 2015;28:1537-41.

17. Steiner LA, Bizzarro MJ, Ehrenkranz RA, Gallagher PG. A decline in the frequency of neonatal exchange transfusions and its effect on exchange-related morbidity and mortality. Pediatrics. 2007;120:27-32.

18. Patra K, Storfer-Isser A, Siner B, Moore J, Hack M. Adverse events associated with neonatal exchange transfusion in the 1990s. J Pediatr. 2004;14:626-31.

19. Sabzehei MK, Basiri B, Shokouhi M, Torabian S. Complications of exchange transfusion in hospitalized neonates in two neonatal 
centers in Hamadan, a five-year experience. J Compr Pediatr. 2015;6:e20587.

20. Spitzer AR, Ellsbury DL, Handler D, Clark RH. The Pediatrix BabySteps $^{\circledast}$ Data Warehouse and the Pediatrix QualitySteps improvement project system-tools for "meaningful use" in continuous quality improvement. Clin Perinatol. 2010;37:49-70.

21. American Academy of Pediatrics Subcommittee on Hyperbilirubinemia. Management of hyperbilirubinemia in the newborn infant 35 or more weeks of gestation. Pediatrics. 2004;114:297-316

22. Olusanya BO, Iskander IF, Slusher TM, Wennberg RP. A decision-making tool for exchange transfusions in infants with severe hyperbilirubinemia in resource-limited settings. J Perinatol. 2016;36:338-41.

23. Olsen IE, Groveman SA, Lawson ML, Clark RH, Zemel BS. New intrauterine growth curves based on United States data. Pediatrics. 2010;125:e214-24.

24. Gunnink SF, Vlug R, Fijnvandraat K, Van Der Bom JG, Stanworth SJ, Lopriore E. Neonatal thrombocytopenia: etiology, management and outcome. Expert Rev Hematol. 2014;7:387-95.

25. Roberts I. Neonatal thrombocytopenia: causes and management. Arch Dis Child Fetal Neonatal Ed. 2003;88:F359-64.

26. Jain A, Agarwal R, Sankar MJ, Deorari A, Paul VK. Hypocalcemia in the newborn. Indian J Pediatr. 2010;77:1123-8.

27. Vemgal P, Ohlsson A. Interventions for non-oliguric hyperkalaemia in preterm neonates. Cochrane Database Syst Rev. 2012; CD005257.
28. Ballabh P. Intraventricular hemorrhage in premature infants: mechanism of disease. Pediatr Res. 2010;67:1-8.

29. Hornik CP, Fort P, Clark RH, Watt K, Benjamin DK, Smith PB, et al. Early and late onset sepsis in very-low-birth-weight infants from a large group of neonatal intensive care units. Early Hum Dev. 2012;88:S69-74.

30. Ramani M, Ambalavanan N. Feeding practices and necrotizing enterocolitis. Clin Perinatol. 2013;40:1-10.

31. Zwiers C, Scheffer-Rath ME, Lopriore E, de Haas M, Liley HG. Immunoglobulin for alloimmune hemolytic disease in neonates. Cochrane Database Syst Rev. 2018;3:CD003313.

32. Yu C, Li H, Zhang Q, He H, Chen X, Hua Z. Report about term infants with severe hyperbilirubinemia undergoing exchange transfusion in Southwestern China during an 11-year period, from 2001 to 2011. PLoS ONE. 2017;12:e0179550.

33. Jackson JC. Adverse events associated with exchange transfusion in healthy and ill newborns. Pediatrics. 1997;99:E7.

34. Figueras-Aloy J, Rodriguez-Miguelez JM, Iriondo-Sanz M, Salvia-Roiges MD, Botet-Mussons F, Carbonell-Estrany X. Intravenous immunoglobulin and necrotizing enterocolitis in newborns with hemolytic disease. Pediatrics. 2010;125:139-44.

35. Platt MJ. Outcomes in preterm infants. Public Health. 2014;128:399-403.

36. Boghossian NS, Geraci M, Edwards EM, Horbar JD. Morbidity and mortality in small for gestational age infants at 22 to 29 weeks' gestation. Pediatrics. 2018;141. pii: e20172533. https:// doi.org/10.1542/peds.2017-2533. 